\title{
Análise De Agrupamento Da ICTIOFAuna Recifal Do Brasil CoM Base EM Dados Secundários: UMa AVAliaÇão Crítica.
}

\author{
Maria Elisabeth de Araújo ${ }^{1,2}$ \\ CAROLINE VIEIRA FEITOSA ${ }^{2}$ \\ ${ }^{1}$ Departamento de Oceanografia, UFPE \\ 2 Pesquisadora do Grupo de Ictiologia Marinha \\ Tropical (IMAT-UFC) \\ betharau@ufpe.br
}

Recebido: 05/06/2003

Aceito: 06/11/2003

\section{RESUMO}

A literatura que trata da diversidade é bastante numerosa e disponibiliza informações fundamentais que podem ser utilizadas como banco de dados para pesquisas com outros enfoques científicos. O presente trabalho reúne 185 espécies de peixes recifais brasileiros retiradas de listas contidas em 16 publicações e propõe uma análise de agrupamento, fornecido através do coeficiente de Jaccard. Os resultados destacam dois grupos principais formados pelas ilhas oceânicas e outro pelas localidades da costa. Com base na interpretação deste agrupamento, as autoras discutem as questões conceituais sobre os estudos zoogeográficos e as metodologias utilizadas para os levantamentos ictiofaunísticos.

Palavras-chave: Diversidade, zoogeografia, análise de agrupamento, peixes recifais, Brasil.

\section{Cluster analysis of brazilian reef fishes based on secondary date: a critical avaliation.}

\begin{abstract}
The bibliography concern diversity is very large, and gives fundamental information that could be used as secondary date for scientific research. This paper uses 185 Brazilian reef fishes took from 16 papers, and propose a cluster analysis by Jaccard coefficient. The result showed two distinct groups: oceanic isles and coastal areas. Based on this cluster analysis, the authors make some concepts considerations about zoogeography and methods to obtain a fish list.
\end{abstract}

Key words: Diversity, zoogeogrphy, cluster analysis, reef fishes, Brazilian reef fishes. 


\section{INTRODUÇÃO}

O conhecimento descritivo já acumulado sobre a diversidade biológica ocupa inúmeras páginas na literatura científica. Alguns cálculos indicam que a diversidade biológica atual representa apenas cerca de $1 \%$ da diversidade produzida ao longo da história. Não seria exagero estimar a diversidade de formas em cerca de $10^{9}$ caracteres individualizáveis para todos os grupos (AMORIM, 1997). O objeto central da sistemática é a diversidade biológica e seus principais problemas são (1) descrever essa diversidade, (2) encontrar um ordenamento subjacente à diversidade, (3) compreender os processos relacionados a essa diversidade e (4) apresentar um sistema geral de referências sobre essa diversidade (AMORIM, 1997).

Um método clássico, do ponto de vista nomotético, de se estudar a diversidade é simplesmente descrevê-la. Existem duas abordagens da ciência para estudar a diversidade biológica: monotética, em que se procuram as relações gerais, e idiográfica, em que a intenção primária é descrever os indivíduos ou eventos individuais (SHORROCKS, 1980). Na diversidade alfa, o número de espécies dentro de uma comunidade (sua riqueza) é resultante da diversificação numa variedade de nichos ecológicos, enquanto a diversidade beta, as espécies estão orientadas ao hábitat, inseridas ao longo de gradientes ambientais (LOWE-Mc-CONNELL, 1999).

As interações ecológicas podem determinar diferenças regionais na diversidade das espécies. O aumento da diversidade das espécies é proporcional à diversidade de hábitats (LEVINTON, 1995). A ictiofauna é diretamente afetada por variáveis físicas que atuam no seu movimento e na captura de seu alimento (VIANNA, 1997). Este autor (1997) cita trabalhos que explicam as variações de comunidades ictiofaunísticas devido às diferenças ambientais e interações tróficas, aos diversos fatores abióticos, à interação competitiva e disponibilidade de alimento, à complexidade ambiental, influência do substrato, ação antrópica etc. Alguns autores admitem que as diferenças encontradas entre ictiofaunas de áreas vizinhas podem ser explicadas por fatores fisiográficos e variação na composição do substrato (VIANNA, 1997). Estudando os peixes que vivem nas lagoas dos atóis da Polinésia francesa, GALZIN et al. (1994) concluem que a diversidade destes animais está significativamente relacionada ao tamanho do atol (perímetro) e à profundidade média e que os fatores que afetam esta diversidade dependem de cada família.

A alta diversidade dos peixes recifais pode estar sendo determinada por fatores extrínsecos, tais como: vicariância, estabilidade climática, heterogeneidade espacial, alta produtividade etc. Os fatores intrínsecos, como a predação e competição, são maiores nos trópicos e podem desencadear eventos de especiação. A predação exerce efeitos opostos sobre os estrategistas-r, selecionando para a uniformidade, enquanto os estrategistas-K são selecionados para a diversidade (LOWE-McCONNELL, 1999).

Os recifes de coral são as maiores estruturas produtoras biologicamente da terra, só comparáveis às densas florestas chuvosas tropicais (MICHAEL, 1998), ambos presentes no Brasil. A alta diversidade em baixas latitudes é uma característica de comunidades de plantas e animais, incluindo os peixes, destacadamente os tropicais que vivem em recifes (LOWE-McCONNELL, 1999). Embora as áreas de recifes de coral atinjam menos de $1 \%$ da área total oceânica, cerca da metade de todas as espécies de peixes marinhos conhecidos estão associados aos recifes. O número total de espécies de peixes recifais está estimado em 7.000 (MICHAEL, 1998).

A ictiofauna recifal do Atlântico Sul Ocidental já foi uma das menos conhecidas, tendo sido ignorada em todas as comparações interoceânicas (FLOETER \& GASPARINI, 2000). Em contrapartida, o Atlântico Ocidental tem sido objeto de muitos estudos faunísticos e zoogeográficos, sendo a ictiofauna mais conhecida no Atlântico Norte, especialmente nas Bahamas, Flórida e Bermudas (BRIGGS, 1974). A homogeneidade faunística do Atlântico Ocidental tropical é

Tropical Oceanography, Recife: v. 31, n. 2, p. 171-192 2003 
questionada com base em fatores físicos e hidrológicos do oceano, quanto ao endemismo (RAMOS, 1994). Até a década de 80, acreditava-se que a ictiofauna recifal brasileira era composta por espécies da fauna do Caribe (MOURA et al., 1999), porém hoje se sabe que muitas espécies da ictiofauna do Atlântico Sul Ocidental são endêmicas, embora similares às espécies do Atlântico Norte Ocidental (FLOETER \& GASPARINI, 2000). A ictiofauna litorânea e parte dos peixes recifais do Brasil e Caribe estão separadas pela maior barreira do Atlântico Sul: o Rio Amazonas (BRIGGS, 1995; FLOETER \& GASPARINI, 2000). A formação da barreira causada pelo Amazonas data de apenas 5 a 6 milhões de anos (HOORN et al. 1995). Este efeito vicariante separou as ictiofaunas do norte e sul do Atlântico Oeste (JOYEUX et al., 2001). Os resultados de RAMOS (1994) para peixes marinhos demersais reforçam a existência de uma descontinuidade entre a fauna do Nordeste brasileiro e do Atlântico Norte tropical.

Uma interpretação alternativa seria que o Rio Amazonas e rios associados, com sua vasta área de influência com águas costeiras de baixa salinidade desde o norte do Ceará, formariam uma área de endemismo justamente pela menor salinidade. Assim a costa entre o norte do Ceará até a Amapá seria a área de maior endemismo do Brasil, não porque o rio Amazonas sirva como barreira para as espécies do Caribe (várias espécies do Caribe ocorrem nas costas nordeste e sudeste do Brasil). O ramo norte da corrente Sul Equatorial seria um candidato melhor para servir como barreira entre a fauna caribenha e brasileira, mas a sua eficácia não é muito grande, já que a maioria das espécies tropicais ocorre desde o sul dos EUA até o sul do Brasil. As coletas de Caridea sugerem que os recifes de corais ao largo do Maranhão abrigariam o maior número de espécies endêmicas do Brasil (CHRISTOFFERSEN, 1980 e com. pess. 2002).

A formação de uma barreira geográfica ou evento vicariante pode proporcionar a formação de novas espécies com distribuição restrita a uma dada região. As comunidades de peixes de recifes de coral variam conforme o hábitat recifal, entre recifes e de uma localidade geográfica a outra, ocorrendo em muitas regiões espécies endêmicas. Nos recifes do Havaí, por exemplo, 24\% das 460 espécies são endêmicas (MICHAEL, 1998). O oeste do Atlântico Sul é caracterizado pela alta taxa de endemismo (18 a 20\%) para os peixes recifais, chegando a 33\% no Brasil, onde 23\% das espécies estão recentemente relacionadas com aquelas do Caribe (LABOREL, 1970; LEÃO, 1983). Metade das espécies endêmicas das ilhas oceânicas tropicais, assim como $74 \%$ das espécies endêmicas da Província Brasileira são bentônicos e de desova demersal, com curto período de duração larval, implicando na dispersão restrita. A taxa de endemismo e essas características biológicas variam de acordo com o isolamento geográfico das ilhas oceânicas do Atlântico Sul (FLOETER \& GASPARINI, 2000).

A fauna de peixes recifais brasileira está representada por cerca de 320 espécies distribuídas pela costa e ilhas oceânicas. Padrões na composição da comunidade de peixes têm causas distintas em larga escala: história evolucionária de diferentes regiões, processos de dispersão e gradientes ambientais básicos (FLOETER et. al., 2001b). As taxas de endemismo no Atol das Rocas e em Fernando de Noronha estão estimadas em 6,9\% e 6,3\%, respectivamente (FLOETER \& GASPARINI, 2000). Trindade tem 14,6\% de peixes endêmicos, sendo um terço (37,4\%) largamente distribuído no Atlântico tropical (FLOETER \& GASPARINI, 2001). Nas ilhas oceânicas, os peixes com desova bentônica são mais freqüentemente endêmicos, enquanto aqueles com desova pelágica são mais especiosos que no continente (FLOETER \& GASPARINI, 2000).

Os peixes associados aos recifes apresentam dois tipos de desova: (1) pelágica, na qual os ovos esféricos flutuantes são dispersos diretamente na coluna d’água; (2) demersal, em que os ovos adesivos, mais pesados que a água do mar, são usualmente cuidados pelos pais até atingirem a fase larval planctônica. A duração do estágio pelágico depende do tipo de desova (THRESHER, 1991), tendo maior duração na reprodução pelágica (GUIMARÃES et al., 2001). A diferença na

Tropical Oceanography, Recife: v. 31, n. 2, p. 171-192 2003 
diversidade da ictiofauna continental e das ilhas oceânicas deve-se tanto a fatores biológicos, como o fato dos peixes recifais serem, em sua maioria, estrategistas-K, quanto a fatores abióticos, como as correntes oceânicas (LOWE-McCONNEL, 1999).

Próximo de Fernando de Noronha e Atol das Rocas, a corrente Sul Equatorial subdivide-se em dois ramos: o ramo norte origina a corrente das Guianas e o ramo sul dá origem à corrente do Brasil (GHERARDI, 1996), que são as principais correntes de superfície da margem continental do Brasil (SILVEIRA et al. 1994). Essas correntes podem ser responsáveis pelo transporte de ovos e larvas, principalmente de peixes, as quais podem colonizar áreas ao longo da costa Norte e Nordeste do Brasil. Acredita-se que o tipo de desova, a área geográfica e as condições oceanográficas são os principais responsáveis pelo sucesso da colonização de peixes em recifes (JOYEUX et al. 2001).

Publicações sobre a ictiofauna recifal brasileira tornaram-se mais evidentes apenas no final da década de 90. Esses trabalhos resultaram em descrição de novas espécies (SAZIMA, et al., 1998; ROCHA \& ROSA, 1999; GOMES, et al., 2000; MOURA et al., 2001; ROCHA \& ROSA, 2001b), levantamentos ictiofaunísticos (ROCHA, et al., 1998; ARAÚJO et al., 2000, GASPARINI \& FLOETER, 2001), distribuição geográfica de espécies (MOURA, et al., 1999; JOYEUX et al., 2001; FLOETER \& GASPARINI, 2000; FLOETER et al., 2001a) e estudos sobre estruturas de comunidade (ROSA \& MOURA, 1997; ROCHA \& ROSA, 2001a).

A presente pesquisa tem como objetivos principais reunir inventários publicados sobre peixes recifais do Brasil, incluindo outros trabalhos das autoras, e elaborar propostas de agrupamentos que baseiem discussões sobre algumas questões sobre diversidade e conceitos a ela atrelados.

\section{MATERIAL E MÉtodos}

Após um levantamento bibliográfico, foram selecionadas dezesseis publicações que tratam de levantamentos ictiofaunísticos brasileiros, com ênfase aos ambientes recifais (Tabela 1). As áreas de estudo incluíram várias localidades da região Nordeste, Rio de Janeiro e ilhas oceânicas. A partir das listagens fornecidas nestes trabalhos, elaborou-se uma lista de espécies de peixes recifais que subsidiasse a análise de agrupamento e a discussão teórica (Anexo 1). Os peixes recifais contidos no inventário das espécies marinhas da Área de Proteção Ambiental (APA) Costa dos Corais (FERREIRA \& CAVA, 2001) foram utilizados como referência, por apresentar o maior número de espécies registradas, para comparação entre as localidades estudadas (Anexo 1). O levantamento ictiofaunístico dos estados da Paraíba, Pernambuco e Alagoas, realizado por KOIKE \& GUEDES (1981), inclui tanto as espécies ditas recifais, como aquelas que vivem em seu entorno; neste caso, foram selecionadas apenas aquelas encontradas em hábitats recifais, segundo descrição dos próprios autores.

A análise de classificação aqui aplicada originou-se de uma matriz de dados binários (presença ou ausência), composta por 185 colunas (espécies) e 11 linhas (locais de amostragens), no Modo Q (agrupando-se as amostragens, ou locais amostrados em função da composição de espécies). Utilizou-se como medida de semelhança (ou índice de similaridade) o coeficiente de Jaccard (1908), cuja fórmula é $\mathrm{Cj}=\mathrm{j} /(\mathrm{a}+\mathrm{b}-\mathrm{j})$, onde j é o número de espécies encontradas nos dois locais, a é o número de espécies encontradas no local “a” e b é o número de espécies encontradas no local "b".

A análise de agrupamento (cluster analysis) foi utilizada para comparar a similaridade entre alguns recifes costeiros e ilhas oceânicas brasileiras. O critério de agrupamento escolhido foi o Método de Ward, que gera grupos com máximo de homogeneidade interna e heterogeneidade inter-

Tropical Oceanography, Recife: v. 31, n. 2, p. 171-192 2003 
grupos, sendo apontado por MILLIGAN \& COOPER (1987) e PEREIRA (1993) como um dos melhores métodos de agrupamento disponíveis. A interpretação foi efetuada por análise visual dos dendogramas e as análises efetuadas através do Pacote de Software NTSYS.

\section{RESUltados}

Os resultados desta pesquisa estão baseados na análise da diversidade de peixes recifais listada a partir de dezesseis publicações científicas (Tabela 1), totalizando 185 espécies, pertencentes a 63 famílias (Anexo 1) e onze localidades brasileiras, incluindo regiões da costa de oito Estados e quatro conjuntos ou ilhas oceânicas.

A análise de agrupamento forneceu o dendograma apresentado na Figura 1. Verifica-se dois grupos distintos: um formada pelas localidades continentais e outro pelas ilhas oceânicas, com coeficientes de Jaccard (CJ) equivalentes a 0,54 e 0,37, respectivamente. Dentro do agrupamento continental, a maior similaridade encontrada está entre as espécies do Rio Grande do Norte e da Paraíba $(\mathrm{CJ} \approx 0,74)$, seguindo às da APA Costa dos Corais $(\mathrm{CJ} \approx 0,65)$. Um outro grupo continental está indicado para os estados da Bahia e Rio de Janeiro $(\mathrm{CJ} \approx 0,64)$. O agrupamento do Ceará e Maranhão apresentou um coeficiente baixo com as demais localidades da costa, algo em torno de 0,56 e 0,54, respectivamente. Bem franca também foi a associação de similaridade da diversidade encontrada para as espécies de peixes listadas para Trindade $(\mathrm{CJ} \approx 0,38)$ e o Arquipélago São Pedro e São Paulo $(\mathrm{CJ} \approx 0,44)$. No entanto, a similaridade da diversidade entre Fernando de Noronha e o Atol das Rocas ficou na ordem de 0,64.

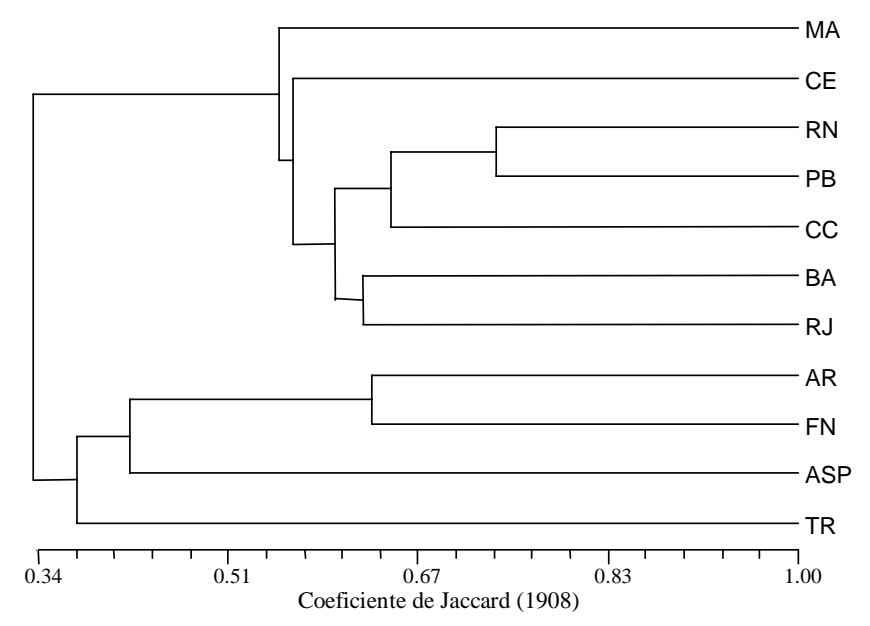

FIGURA 1: Análise de agrupamento, com base no coeficiente de Jaccard, para a diversidade de espécies de peixes recifais do Brasil.

Legenda: Maranhão (MA), Ceará (CE), Rio Grande do Norte (RN), Paraíba (PB), Costa dos Corais (CC), Bahia (BA), Rio de Janeiro (RJ), Atol das Rocas (AR), Fernando de Noronha (FN), Arquipélago São Pedro e São Paulo (ASP) e Trindade (TR). 


\section{DISCUSSÃO}

Comparando o dendograma aqui encontrado com aquele elaborado por FLOETER e GASPARINI (2000), que utilizaram coeficientes de Sorensen para diversas localidades do Atlântico Sudoeste, pode-se verificar que, igualmente, a formação de dois grupos principais: um continental e outro para as ilhas oceânicas para peixes recifais. Do mesmo modo, o agrupamento entre as ilhas oceânicas também coincidiu com a topografia aqui obtida, embora a natureza dos dados tenha sido diferente: diversidade de espécies (na presente pesquisa) versus riqueza de espécies e diversidade de famílias (op. cit., 2000). Segundo estes autores (2000), a riqueza de espécies que ocorrem no Nordeste (Paraíba) é mais similar àquela encontrada no Sudeste (Três Ilhas), que entre a Paraíba e as ilhas oceânicas do Nordeste (Fernando de Noronha e Atol das Rocas, por exemplo), cuja distância é menor. A diversidade costuma ser maior na costa, diminuindo à proporção que aumenta a distância entre as ilhas e o continente (JOYEUX et al., 2001).

Algumas outras espécies têm distribuição restrita à costa ou a certas ilhas oceânicas. É o caso dos acanturídeos, mais especificamente Acanthurus chirurgus que está presente na costa brasileira, em Fernando de Noronha e no Atol das Rocas, mas não consta das listas para Trindade (GASPARINI \& FLOETER, 2001) e para o Arquipélago São Pedro e São Paulo (LUBBOCK \& EDWARDS, 1981). Neste Arquipélago, nenhuma espécie de acanturídeos foi encontrada até o momento (JOYEUX et al., 2001). Espécies da família Lutjanidae estão bem representadas na costa do Atlântico Ocidental, mas é pouco abundante em número de espécie por família em certas ilhas oceânicas (FLOETER \& GASPARINI, 2000). Lutjanus chrysurus e L. purpureus, por exemplo, estão registradas apenas para Fernando de Noronha (FLOETER et al. 2001a), enquanto Lutjanus cf. vivanus, para Trindade (GASPARINI \& FLOETER, 2001). Apenas nesta ilha, incluindo a costa e demais ilhas oceânicas brasileiras estudadas, ainda não foi verificada a ocorrência de Lutjanus jocu (op. cit., 2001). Haemulidae e Lutjanidae, embora sejam famílias com um número representativo de espécies nos recifes costeiros brasileiros, estão pobremente ou não são registradas nas ilhas oceânicas (FLOETER et. al., 2001).

A primeira dificuldade ao se realizar um trabalho teórico como este é a padronização das informações contidas na literatura. Por vezes dispõe se apenas de listas que incluem diversos hábitats marinhos, abrangendo grande parte dos ambientes recifais, por vezes listas que tratam dos peixes encontrados nos recifes, mas estão presentes várias espécies tipicamente costeiras ou estuarinas, porém que vivem em volta dos recifes. Analisando o Anexo 1, pode-se perceber que, das 193 registradas para os recifes brasileiros, apenas para os Estados de Pernambuco e Alagoas, Costa dos Corais, (KOIKE \& GUEDES, 1981 e FERREIRA \& CAVA, 2001) e Paraíba (op. cit., 1981) ocorreram espécies exclusivas destas regiões. Espécies de linguados, como Bothus ocellatus e Symphurus plagusia, são estuarinas e não chegam a depender diretamente dos ambientes recifais, contradizendo, em parte, a classificação de KOIKE \& GUEDES, 1981. Centropomus parallelus, Netuma barba (CERVIGÓN et al., 1992, NAKAMURA et al., 1986) Bairdiella ronchus, Elops saurus e espécies de Eugerres (VASCONCELOS-FILHO \& OLIVEIRA, 2000), também não podem ser consideradas recifais, assim como Gymnura micrura, que vive junto ao fundo da plataforma continental.

A ausência de registro para uma única localidade pode se dever tanto a fatores biológicos e ambientais, quanto a problemas de insuficiência de amostragem. Nos demais inventários aqui estudados (Anexo 1), algumas espécies estão ausentes apenas para uma dada região. Não estão registradas, por exemplo, Chaetodon striatus para o Atol das Rocas (ROSA \& MOURA, 1995); Carangoides bartholomaei, Sparisoma frondosum para Trindade 
(GASPARINI \& FLOETER, 2001); Gymnothorax moringa, Malacanthus plumieri, Anisotremus surinamensis, Pseudupneus maculatus, Acanthurus bahianus e A. coeruleus para o Arquipélago São Pedro e São Paulo (LUBBOCK \& EDWARDS, 1981), Apogon americanus, Sparisoma frondossum e S. radians para os recifes costeiros do Ceará (ARAÚJO et al., 2000); Kyphosus sectatrix para o Rio de Janeiro (FERREIRA et al., 2001).

Para as ilhas oceânicas é de se esperar uma menor diversidade em relação à costa continental, pois a ausência de algumas espécies pode ser explicada pelo pequeno tamanho das ilhas, o que limita o assentamento de novos indivíduos em hábitats disponíveis (JOYEUX et al., 2001). No entanto, problemas de identificação ao nível de espécie também podem ocorrer para algumas espécies, como no caso daquelas do gênero Sparisoma, segundo exemplos acima citados. Além dos problemas de sinonímia e mudanças taxonômicas causadas pelas revisões sistemáticas, verificadas nos trabalhos mais antigos, a utilização de bibliografia estrangeira e o desconhecimento da distribuição em áreas pioneiramente estudadas, podem incorrer no erro de ampliar a distribuição. Eupomacentrus leucostictus, registrada por KOIKE \& GUEDES (1981) para Pernambuco, ilustra bem o que está tratado acima: o gênero agora é Stegastes e a citação de $S$. leucostictus deve ser, provavelmente, um registro com base na distribuição ampliada das espécies que ocorrem no Caribe (BÖHLKE \& CHAPLIN apud ROSA et al. 2002).

$\mathrm{O}$ entendimento sobre as comunidades de peixes recifais está baseado em dados parciais que, geralmente, podem não estar corretos (SALE, 1991). Como as fontes de dados das listas publicadas não são as mesmas, nem o levantamento das ictiofaunas se encontra estudado satisfatoriamente, resultados diferentes são esperados. O número insuficiente de censos para inventariar uma dada área também pode mascarar os resultados quando se faz uma análise de agrupamento (op. cit., 1991). As incongruências encontradas nas análises de agrupamento da diversidade de peixes recifais são esperadas, pois os dados disponíveis na literatura científica para este tipo de classificação ainda são insuficientes (LOWE-Mc-CONNELL, 1999). Por exemplo, nos recifes existem cerca de 400 espécies de peixes, sendo mais de 50 endêmicas e descoberta apenas nos últimos 20 anos (GUIMARÃES et al. 2001). Outra citação ilustrativa para o Brasil é MOURA et al. (1999), quando afirmam que a maior riqueza de espécies de peixes recifais (estimada preliminarmente em 150 espécies) deve ser aquela encontrada no Arquipélago de Abrolhos, embora poucos peixes estejam colecionados.

Quando se aborda a questão da diversidade biológica é necessário ter em mente a existência de dois aspectos distintos, mas relacionados entre si: o número de grupos diferentes destas entidades que, poderão ou não, ser compartilhados pelos grupos (AMORIM, 1997). Esta dificuldade pode ser percebida também quando se tenta fazer uma análise de agrupamento a partir de dados de diversidade. O primeiro impasse começa com uma antiga e polêmica questão: o conceito de espécie! Decorrem deste outros igualmente polêmicos: sub-espécies, especiação e endemismo. Além da questão conceitual, existem problemas nas questões práticas taxonômicas; como a padronização dos nomes científicos ou de metodologias; como a seleção de espécies descartadas para a análise ou agrupadas por não terem sido diferenciadas em campo.

A recomendação de SALE \& DOUGLAS (1981): excluir algumas espécies amostradas por censo visual, tem sido adotada por vários pesquisadores. VIANNA (1997) retirou nove espécies de peixes como procedimento alternativo para minimizar erros amostrais do censo visual; ROSA \& MOURA (1997) afirmam que “a diversidade encontrada em Rocas é menor que aquela descrita para a Província do Índico-Oeste, mesmo com a exclusão das espécies noturnas, pequenas e crípticas de suas análises”. Nos dados aqui apresentados para o Rio de

Tropical Oceanography, Recife: v. 31, n. 2, p. 171-192 2003 
Janeiro, FERREIRA et al. (2001) descartaram os peixes que formam grandes cardumes, acima de 500 indivíduos, por pesarem na contagem e devido à casualidade de sua ocorrência.

Os trabalhos utilizados nesta pesquisa (Tabela 1) estão baseados, na maioria, em método do censo visual, que vem sendo altamente difundido (BOHNSACK \& BANNEROT, 1986, BORTONE et al., 1989) e aceito no meio científico por ser não destrutivo e eficiente (ROSA \& MOURA, 1997), além de prático e barato. Como os demais métodos de inventário, o censo visual não reflete toda a ictiofauna e apresenta algumas limitações. VIANNA (1997) avalia diversos trabalhos que discutem este método e concluem que ele subestima as espécies raras, noturnas, crípticas, pequenas e superestima as espécies que formam cardumes e que são territorialistas. O investigador também pode ocasionar erros nos censos por falta de segurança na identificação de algumas espécies ou preferência pessoal por outras. As replicações dos censos e a periodicidade das amostragens fazem decrescer o erro desse método.

GALZIN et al. (1994) afirmam que a determinação de parâmetros utilizados para relacionar área e diversidade de espécies marinhas necessita de mais estudos. Estudos sistemáticos adicionais, incluindo as relações filogenéticas dos táxons poderão elucidar questões sobre o conhecimento das relações biogeográficas entre as diversas áreas nas costas ocidental e oriental das Américas (RAMOS, 1994). Como a grande maioria dos peixes recifais está diretamente associada às estruturas dos próprios recifes, o estudo de eventos evolutivos fará compreender a diversidade dessa ictiofauna. A ação antrópica também pode causar mudanças no ambiente e afetar as estruturas populacionais de peixes que vivem numa dada área, ocasionando migração ou favorecendo a apenas algumas espécies (STEPHENS et al., 1988 apud VIANNA, 1997).

Concorda-se absolutamente com FERREIRA et al. (2001) quando concluem que a produção de conhecimento básico, em termos de abundância e diversidade, é fundamental para desenvolver modelos que possam se acoplados e melhores compreendidos para a preservação dessas áreas.

\section{Agradecimentos}

Aos Professores Dr. Petrônio Alves Coelho (UFPE) e Dr. Martin L. Christoffersen (UFPB), pelas sugestões e leitura do manuscrito, e ao Dr. Nonato da Conceição (LABOMAR), por ter fornecido o programa de análise de agrupamento.

\section{REFERÊNCIAS BIBLIOGRÁFICAS}

AMORIM, D. S. Elementos básicos de Sistemática Filogenética, Ed. Holos, 2ª . ed., Ribeirão Preto, SP, 275p., 1997.

ARAÚJO, M. E. et al. Ictiofauna marinha do estado do Ceará, Brasil: II. Elasmobranchii e Actinopiterygii de Arrecifes de arenito da região entre marés. Arq. Ciên. Mar, Fortaleza, v.33, p.133-138, 2000.

BELL, J. D. \& GALXIN, R. Influence of live coral on coral-reef fish communities. Mar. Ecol. Prog. Ser., v.15, p.265-274, 1984. 
BOHNSACK, J. A. \& BANNEROT, S. P. A stationary visual census technique for quantitatively assessing community structure of coral reef fishes. NOAA Technichal Report, v.41, p.1-15, 1986.

BORTONE, S. A.; HASTINGS, R. W. \& OGLESBY, J. L. Quantification of reef fish assemblages: A comparison of three methods for visually assessing reef fish communities: time and area compensated. Northeast Gulf Sci., v.10, n.2, p.85-96, 1989.

BRIGGS, J.C. Marine zoogeography. Mc-Graw-Hill Book Co., New York, 475p., 1974.

BRIGGS, J.C. Global biogeography. Developments in paleontology and stratigraphy. Elsevier, Amsterdam, v.14, 1995.

CERVIGÓN, F. et al. Fichas FAO de identificación de especies para los fines de la pesca. Guia de campo de las especies comerciales marinas y de aquas salobres de la costa septentrional se Sur América. FAO, Roma, 519p., 1992.

COELHO, P. A. et al. Alguns peixes ornamentais ocorrentes no litoral do Município do Rio Formoso, PE. Bol. Téc. Cient. CEPENE, Tamandaré (PE), v 1, n 1, p.103-127, 1993.

CORREIRA, L.B. Ictiologia marinha do Parque Estadual Marinho Pedra da Risca do Meio, Fortaleza, CE. Monografia apresentada no Departamento de Engenharia de Pesca da UFC, 33p. 2002.

EDWARDS, A. \& LUBBOCK, R. Marine zoogeography of St Paul's Rocks. J. Biogeogr. ,v.10, p.65-72, 1983.

FEITOZA, B. M. Composição e estrutura da comunidade de peixes recifais da Risca do Zumbi, Rio Grande do Norte. Dissertação (Mestrado em Zoologia), Universidade Federal da Paraíba, João Pessoa, 156p., 2001.

FERREIRA, B. P. \& CAVA, F. Ictiofauna marinha da APA Costa dos Corais: Lista de espécies através de levantamento da pesca e observações subaquáticas. Bol. Tecn. Cient. CEPENE, Tamandaré (PE), v.9, n.1, p.167-180, 2001.

FERREIRA, B. P., MAIDA, M. \& SOUZA, A. E. T. Levantamento inicial das comunidades de peixes recifais da região de Tamandaré - PE. Bol. Técn. Cient. CEPENE, Tamandaré (PE), v.3, n.1, p.211-230, 1995.

FERREIRA, C. E. L., GONÇALVES, J. E. A. \& COUTINHO, R. Community structure of fishes and habitat complexity on a tropical rocky shore. Env. Biol. Fishes, v. 61, p.353-369, 2001.

FLOETER, S. R. \& GASPARINI, J. L. The southwestern Atlantic reef fish fauna: composition and zoogeographic patterns. J. Fish Biol., v.56, p.1099-1114, 2000.

FLOETER, S. R. \& GASPARINI, J. L. Brazilian endemic reef fishes. Coral reefs, v.19, p.292, 2001. 
FLOETER, S. R.; GASPARINI, J. L.; ROCHA, L. A.; FERREIRA, C. E. L; CARLOS, A. R.; FEITOZA, B. M.; NUNAN, G. W. Brazilian reef fish fauna: checklist and remarks. Biobase Project: www. biobase. org/BCF/Index.html, 2001a.

FLOETER, R. S.; GUIMARÃES, R. Z. P.; ROCHA, L. A.; FERREIRA, C. E. L.; RANGEL, C. A.; GASPARINI, J. L. Geographic variation in reef-fish assemblages along the Brazilian coast. Global Ecology \& Biogeography n. 10, p. 423-431, 2001 b.

GALZIN, R. et al. Variation in diversity of coral reef fish between French Polynesian atolls. Coral Reefs, v.13, p.175-180, 1994.

GASPARINI, J. L. \& FLOETER, S. R. The shore fishes of Trindade Island, western South Atlantic. J. Nat. Hist., v.35, n.11, p.1639-1656, 2001.

GHERARDI, D. F. M. Recent carbonate sedimentation on the coralline-algal Atol das Rocas, equatorial south Atlantic, Brazil, 1996. Tese (Doutorado em Geologia)- Department of Geology Royal Holloway, University of London. London.

GOMES, U. L., ROSA, R. S. \& GADIG, O. B. F. Dasyatis marianae sp. n.: a new specie of stingray (Chondrichthyes: Dasyatidae) from the southwestern Atlantic. Copeia, n.2, p.510-515, 2000.

GUIMARÃES, R. Z. P. et al. Peixes recifais: uma riqueza a conhecer. Ciência Hoje v.28, n.168, 2001.

HOORN,C. et al. Andean tectonics as a cause for changing drainage patterns in Miocene northern South America. Geology , v.23, p.237-240, 1995.

JACCARD, P. Nouvelles recherches sur la distribution florale. Bull. Soc. Scie. Nat., v.44, p.223270, 1908.

JOYEUX, J. C. et al. Biogeography of tropical reef fishes: the South Atlantic puzzle. J. Biogeogr., v.28, n.7 p.831-842, 2001.

KoIKe, J. \& Guedes, D. S. Peixes dos arrecifes de Pernambuco e estados vizinhos. In: III Encontro de Zoologia do Nordeste. Anais, Recife (PE), p.35-82, 1981.

LABOREL, J. Les pleupements des madréporaires des côtes tropicales Do Brési. Annals of the University of Abidjan, Series E, v.2, p.1-260, 1970.

LEÃO, Z.M.A.N. Abrolhos - o refúgio pleistocênico de uma fauna terciária de corais. Ciências da Terra, v.8, p.22-24, 1983.

LEVINTON, J.S. Marine Biology: Function, Biodiversity, Ecology. Oxford University Press, New York, 420p., 1995. 
LOPES, P. R. D. et al. Contribuição ao conhecimento da ictiofauna da praia de Itapema, Santo Amaro da Purificação, Baía de Todos os Santos, Bahia. Acta Biol. Leopoldensia, v.21, n.1, p.99105, 1999a.

LOPES, P. R. D., OLIVEIRA-SILVA, J. T. \& SILVA, I. S. Registros adicionais para a ictiofauna da praia de Itapema, Santo Amaro da Purificação, Baía de Todos os Santos, Bahia, com notas sobre a alimentação de jovens de Epinephelus itajara (TELEOSTEI: SERRANIDAE). LECTA, Bragança Paulista, v.17, n.2, p.37-41, 1999b.

LOWE-McCONNEL, R. H. Estudos ecológicos de comunidades de peixes tropicais. Trad. Vazzoler A. E. A., Agostinho, A. A., Cunnhigham, P. T. M. Título original: Ecological Studies in tropical fish communities, São Paulo: EDUSP, 382p., 1999.

LUBBOCK, R. \& EDWARDS, A. The fishes of Saint Paul’s Rocks. J. Fish Biol., v.18, p.135-157, 1981.

MAIDA, M. \& FERREIRA, B. P. Coral Reefs of Brazil: an overview. Proceedings of the $\mathbf{8}^{\text {th }}$ International Coral Reef Symposium v.1, p.263-274, 1997.

MICHAEL, S.W. Reef fishes: a guilde to their identification, behavior, and captive care. T.F.H. public, Inc., New Jersey, 624p., 1998.

MOURA, R. L. Atividade, distribuição e táticas alimentares de uma comunidade de peixes do Atol das Rocas. Dissertação ( Mestrado em Zoologia) - Museu de Zoologia, Departamento de Zoologia, Instituto de Biociências. Universidade de São Paulo, São Paulo, 108p., 1998.

MOURA, R. L., FIGUEIREDO, J. L., SAZIMA, I. A new parrotfish (Scaridae) from Brazil, and revalidation of Sparisoma amplum (Ranzani, 1842), Sparisoma frondosum (Agassiz, 1831), Sparisoma axillare (Steindachner, 1878) and Scarus trispinosus (Valenciennes, 1840). Bull. Mar. Scie., v.68, n.3, p.505-524, 2001.

MOURA, R. L., GASPARINI, J. L. \& SAZIMA, I. New records and range extensions of reef fishes in the Western South Atlantic, with comments on reef fish distribution along the Brazilian coast.

Revta bras. Zool., v.16, n.2, p.513-530, 1999.

NAKAMURA, I. et al. Important fishes trawled off Patagonia. Japan Marine Fishery Resource Research Center, Tokyo, 369p. 1986.

RAMOS, R. T. C. Análise da composição e distribuição da fauna de peixes demersais da plataforma continental da Paraíba e Estados vizinhos. Rev. Nordest. Biol., João Pessoa (PB), v.9, n.1, p.1-30, 1994.

ROCHA, L. A. \& ROSA, I. L. New species of Haemulon (Teleostei: Haemulidae) from the Northeastern brazilian coast. Copeia, n.2, p.447-452, 1999.

ROCHA, L. A. \& ROSA, I. L. Baseline assessment of reef fish assemblages of Parcel Manuel Luiz Marine State Park, Maranhão, north-east Brazil. J. Fish Biol., v.58, p.985-998, 2001a.

Tropical Oceanography, Recife: v. 31, n. 2, p. 171-192 2003 
ROCHA, R.L. \& ROSA. R. S. Halichoeres brasiliensis (Bloch, 1791), a valid wrasse species (Teleostei: Labridae) from Brazil, with notes on the Caribbean species Halichoeres radiatus (Linnaeus, 1758). Aqua, J. Ichth., v.4, n.4, p.161-166, 2001 b.

ROCHA, L. A., ROSA, I. L. \& ROSA, R. S. Peixes recifais da costa da Paraíba, Brasil. Revta bras. Zool., v.15, n.2, p.553-566, 1998.

ROSA, R. S. \& MOURA, R. L. Community structure of reef fishes in the biological reserve of Atol das Rocas, off Brazil. Canada. In: Abstracts, A. S. I. H. annual meeting, Edmonton, Canada, 1995.

ROSA, R. S. \& MOURA, R. L. Visual assessment of reef fish community structure in the Atol das Rocas Biological Reserve, off Northeastern Brazil. Proceedings of the $8^{\text {th }}$ International Coral Reef Symposium v.1, p.983-986, 1997.

SALE, P.F. Perception, pattern, change and the structure of reef fish communities. Env. Biol. Fish. v.21, p.3-15, 1988.

SALE, P. F. The Ecology of Fishes on Coral Reefs. Academic Press: San Diego, 754p, 1991.

SALE, P.F. \& DOUGLAS, W.A. Precision and accuracy of visual census technique for fish assemblages on coral patch reefs. Env. Biol. Fish., v.6, n.3/4, p.33-339, 1981.

SAZIMA, I., GASPARINI, J.L. \& MOURA, R. L. Gramma brasiliensis, a new basslet from the western South Atlantic (Perciformes: Grammatidae). J. Ichth. Aquatic Biol., v.3, p.39-43, 1998.

SHORROCKS, B. A origem da diversidade: as bases genéticas da evolução. Tradução de J. Morgante e P. G. Otto. EDUSP, São Paulo, 181p., 1980.

SILVEIRA, I. C. A. DA MIRANDA, L. B. \& BROWN, W. S. On the origins of the North Brazil Current. J. Geophys. Res., v.99, n.C11, p.501-512, 1994.

SNEATH, P. H. A. \& SOKAL, R. R. Numerical Taxonomy. Freeman, San Francisco, 1973.

THRESHER, R. E. Geographic variability in the ecology of coral reef fishes: evidence, evolution and possible implications. In: The ecology of fishes on coral reefs. Academic Press: San Diego, p.401-436, 1991.

VASCONCELOS-FILHO, A.L. \& OLIVEIRA, A.M.E., Ictiofauna. In: Gerenciamento participativo de estuários e manguezais. Editores Barros, H.M., Macedo, S.J., Leça, E.E., Lima, T. Recife, Ed. Universitária da UFPE, Cap.11, p.143-154, 2000.

VAZZOLER, A. E.; SOARES, L. S. H. \& CUNNINGHAM, P. T. M. Ictiofauna da costa brasileira. In: Lowe-McConnel, R. H. Estudos ecológicos de comunidades de peixes tropicais. São Paulo: Editora da Universidade de São Paulo. p.424-468, 1999.

Tropical Oceanography, Recife: v. 31, n. 2, p. 171-192 2003 
VIANNA, M. Fhysiographic influence in the structure of the suprabenthic fish assemblage in Rocky Shores of Arraial do Cabo, Rio de Janeiro, Brazil. B. Inst. Pesca, v.24, p.25-35, 1997. 
TABELA 1: RELAÇÃO DAS REFERÊNCIAS BIBLIOGRÁFICAS QUE FORNECERAM AS LISTAS DA ICTIOFAUNA, COM AS LOCALIDADES E COORDENADAS DAS ÁREAS PESQUISADAS.

\begin{tabular}{|c|c|c|c|}
\hline REFERÊNCIAS BIBLIOGRÁFICAS & LOCALIDADES PESQUISADAS & ESTADOS & COORDENADAS \\
\hline Rocha \& Rosa (2001a) & Parcel Manuel Luiz & Maranhão & $0^{\circ} 52^{\prime} \mathrm{S} ; 44^{\circ} 15^{\prime} \mathrm{W}$ \\
\hline Feitosa (2002) e Feitoza (2001) & Parrachos de Maracajaú; Risca do Zumbi & Rio Grande do Norte & $5^{\circ} \mathrm{S} ; 35^{\circ} \mathrm{W}$ \\
\hline Araújo et al. (2000) & Flexeiras & Ceará & 3¹3'14"S;39¹7'60"W \\
\hline Araújo et al. (2000) & Pecém & Ceará & $3^{\circ} 32^{\prime} 08^{\prime \prime S} ; 38^{\circ} 48^{\prime} 07^{\prime \prime} \mathrm{W}$ \\
\hline Araújo et al. (2000) & Mucuripe & Ceará & $3^{\circ} 42^{\prime} 09^{\prime \prime} \mathrm{S} ; 38^{\circ} 28^{\prime} 06^{\prime \prime} \mathrm{W}$ \\
\hline Correa (2002) & $\begin{array}{l}\text { Parque Estadual Marinho Pedra da Risca } \\
\text { do Meio }\end{array}$ & Ceará & $\begin{array}{l}3^{\circ} 34^{\prime} 20^{\prime \prime} \mathrm{S} ; 38^{\circ} 26^{\prime} \mathrm{W} ; 3^{\circ} 26^{\prime} \mathrm{S} ; 38^{\circ} 3 \\
3^{\circ} 36^{\prime} \mathrm{S} ; 38^{\circ} 21^{\prime} 60^{\prime \prime} \mathrm{W} ; 3^{\circ} 34^{\prime} 20^{\prime \prime} \mathrm{S} ; 38 \\
\mathrm{~W}\end{array}$ \\
\hline Joike \& Guedes (1981), Rocha et al. (1998) & Litoral da Paraíba & Paraíba & $7^{\circ} \mathrm{S} ; 34^{\circ} 50 \mathrm{~W}$ \\
\hline $\begin{array}{l}\text { Joike \& Guedes (1981), Coelho et al. (1993), } \\
\text { Ferreira et al. (1995a) e Ferreira \& Cava (2001) }\end{array}$ & APA Costa dos Corais & $\begin{array}{l}\text { Pernambuco e } \\
\text { Alagoas }\end{array}$ & $\begin{array}{l}8^{\circ} 42^{\prime} 16^{\prime \prime S} ; 35^{\circ} 04^{\prime} 40^{\prime \prime} \mathrm{W} ; 8^{\circ} 47^{\prime} 44^{\prime \prime} \\
0^{\prime \prime} \mathrm{W} \\
9^{\circ} 46^{\prime} 30^{\prime \prime} \mathrm{S} ; 35^{\circ} 25^{\prime} \mathrm{W} ; 9^{\circ} 32^{\prime} 51^{\prime \prime S} \mathrm{~S} 3 \\
\text { W }\end{array}$ \\
\hline $\begin{array}{l}\text { Lopes et al (1999a,b), Araújo, M.E (com. pess.) } \\
\text { Floeter et al. (2001a) e Moura et al. (1999) }\end{array}$ & Itapema, Abrolhos & Bahia & $12-16^{\circ} \mathrm{S} ; 12^{\circ} 42^{\prime} \mathrm{S} ; 38^{\circ} 45 \mathrm{~W} ; 17^{\circ} 20^{\prime}$ \\
\hline Ferreira et al. (2001) e Floeter et al. (2001a) & Arraial do Cabo e Ilha Grande & Rio de Janeiro & $23^{\circ} \mathrm{S} ; 42^{\circ} \mathrm{W} ; 23^{\circ} 05 \mathrm{~S} ; 44^{\circ} 20 \mathrm{~W}$ \\
\hline Rosa \& Moura (1995) e Floeter et al. (2001a) & Atol das Rocas & Rio Grande do Norte & $3^{\circ} 50^{\prime} \mathrm{S} ; 33^{\circ} 49^{\prime} \mathrm{W}$ \\
\hline $\begin{array}{l}\text { Araújo, M. E (com.pess.) e Floeter et al. } \\
(2001 \mathrm{a})\end{array}$ & Fernando de Noronha & Pernambuco & $3^{\circ} 51^{\prime} \mathrm{S} ; 32^{\circ} 26^{\prime} \mathrm{W}$ \\
\hline Lubbock \& Edwards (1981) & Arquipélago de São Pedro e São Paulo & Rio Grande do Norte & $29^{\circ} 20^{\prime} 33^{\prime \prime W, 0 \circ 55^{\prime} 10^{\prime \prime}}$ \\
\hline $\begin{array}{l}\text { Gasparini \& Floeter (2001) e Floeter et al. } \\
\text { (2001a) }\end{array}$ & Trindade & Espírito Santo & $20^{\circ} 30^{\prime} \mathrm{S} ; 29^{\circ} 20^{\prime} \mathrm{W}$ \\
\hline
\end{tabular}




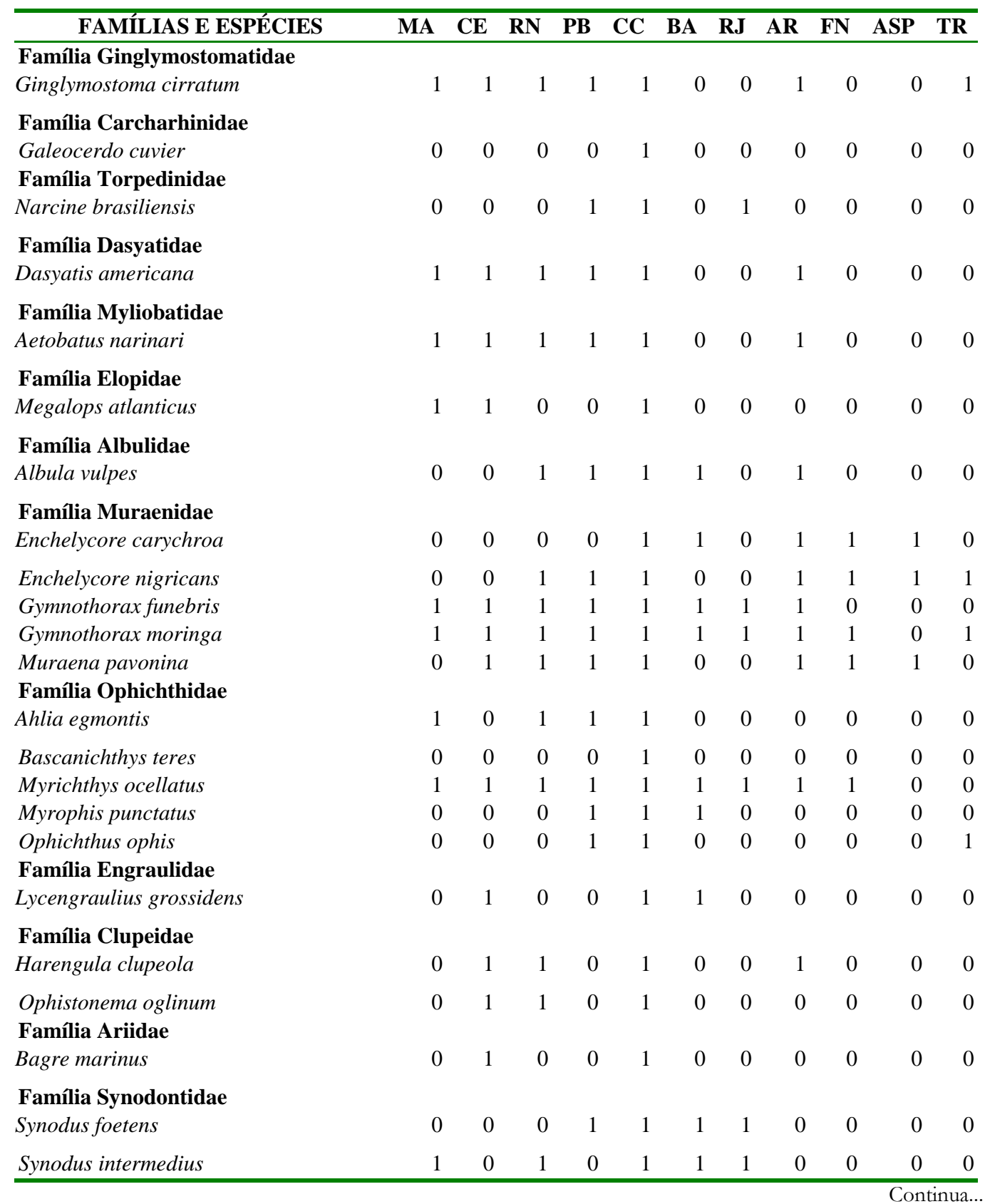


ANEXO 1: REGISTROS BIBLIOGRÁFICOS DA ICTIOFAUNA RECIFAL POR ESTADOS E ILHAS OCEÂNICAS DO BRASIL. Continuação

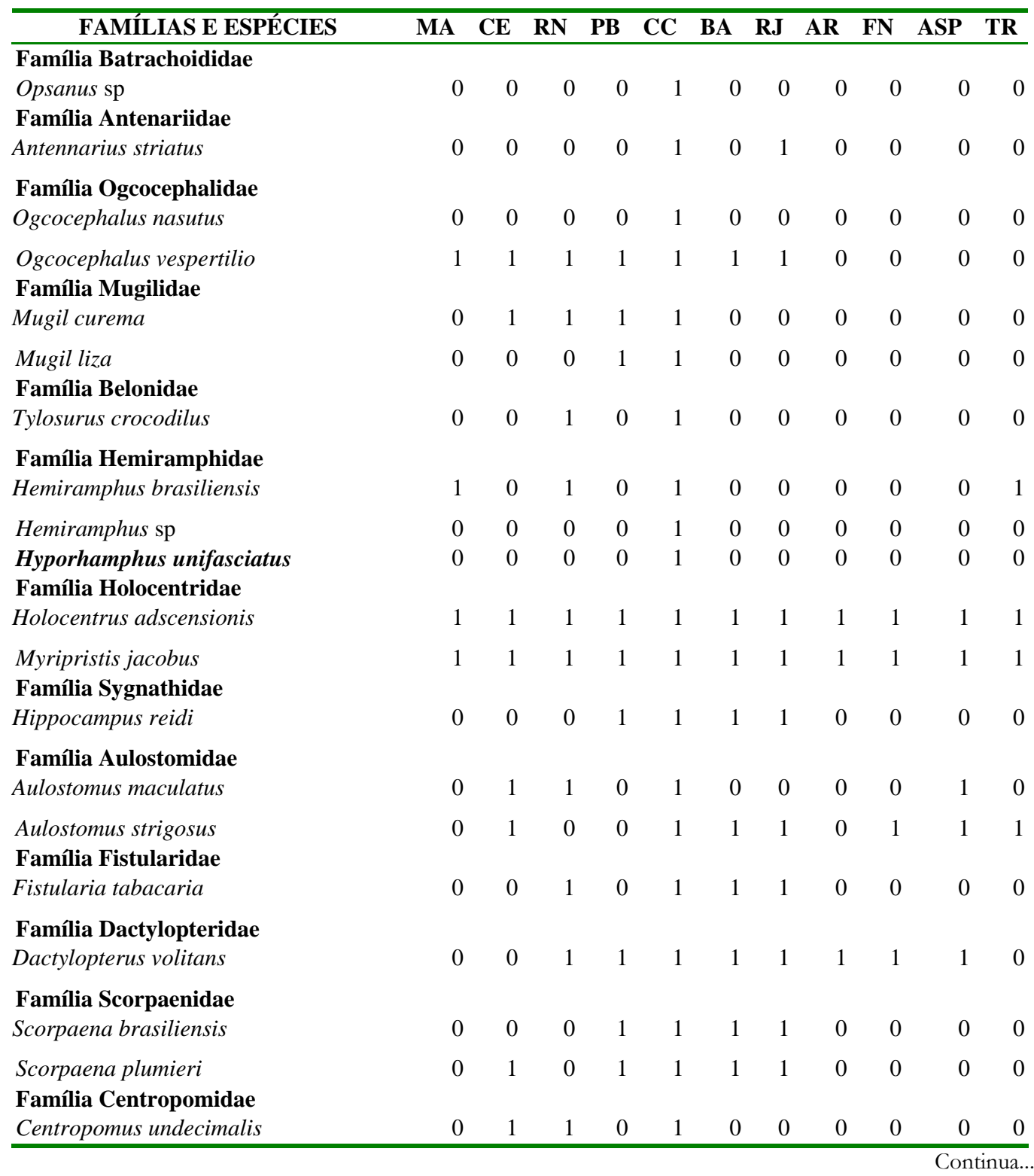


ANEXO 1: REGISTROS BIBLIOGRÁFICOS DA ICTIOFAUNA RECIFAL POR ESTADOS

E ILHAS OCEÂNICAS DO BRASIL. Continuação

\begin{tabular}{lccccccccccr}
\hline \multicolumn{1}{c}{ FAMÍLIAS E ESPÉCIES } & MA & CE & RN & PB & CC & BA & RJ & AR & FN & ASP & TR \\
\hline Família Serranidae & & & & & & & & & & & \\
Alphestes afer & 0 & 0 & 1 & 1 & 1 & 1 & 1 & 0 & 0 & 0 & 0 \\
Cephalopholis fulva & 1 & 1 & 1 & 1 & 1 & 1 & 1 & 1 & 1 & 1 & 1 \\
Diplectrum formosum & 1 & 0 & 0 & 1 & 1 & 0 & 1 & 0 & 0 & 0 & 0 \\
Epinephelus adscensionis & 0 & 1 & 1 & 1 & 1 & 0 & 0 & 0 & 0 & 0 & 1 \\
Epinephelus itajara & 1 & 1 & 1 & 1 & 1 & 1 & 1 & 0 & 0 & 0 & 0 \\
Epinephelus niveatus & 0 & 0 & 0 & 0 & 1 & 0 & 1 & 0 & 0 & 0 & 1 \\
Mycteroperca bonaci & 1 & 1 & 1 & 1 & 1 & 1 & 1 & 1 & 1 & 0 & 1 \\
Mycteroperca interstitialis & 0 & 0 & 1 & 0 & 1 & 1 & 1 & 0 & 0 & 0 & 1 \\
Mycteroperca tigris & 0 & 0 & 0 & 0 & 1 & 0 & 1 & 0 & 0 & 0 & 0 \\
Mycteroperca venenosa & 1 & 0 & 0 & 0 & 1 & 1 & 1 & 0 & 0 & 0 & 1 \\
Paranthias furcifer & 0 & 1 & 1 & 0 & 1 & 0 & 1 & 1 & 1 & 0 & 0 \\
Rypticus saponaceus & 1 & 1 & 1 & 1 & 1 & 1 & 1 & 1 & 1 & 1 & 1 \\
Rypticus subbifrenatus & 0 & 0 & 1 & 1 & 1 & 1 & 0 & 0 & 0 & 0 & 0 \\
Serranus baldwini & 1 & 0 & 0 & 0 & 1 & 0 & 0 & 0 & 0 & 0 & 0 \\
Serranus flaviventris & 1 & 1 & 1 & 1 & 1 & 1 & 1 & 0 & 0 & 0 & 0 \\
Família Grammatidae & & & & & & & & & &
\end{tabular}

Gramma brasiliensis

Família Priacanthidae

Priacanthus arenatus

Família Apogonidae

Apogon americanus

Astrapogon spp

Phaeoptyx pigmentaria

Família Malacanthidae

Malacanthus plumieri

Família Echeneidae

Remora sp

$\begin{array}{lllllllllll}0 & 0 & 0 & 0 & 1 & 0 & 0 & 0 & 0 & 0 & 0\end{array}$

Família Rachycentridae

Rachycentron canadum

$\begin{array}{lllllllllll}1 & 1 & 1 & 1 & 1 & 1 & 0 & 0 & 0 & 0 & 0\end{array}$ 
ANEXO 1: REGISTROS BIBLIOGRÁFICOS DA ICTIOFAUNA RECIFAL POR ESTADOS

E ILHAS OCEÂNICAS DO BRASIL. Continuação

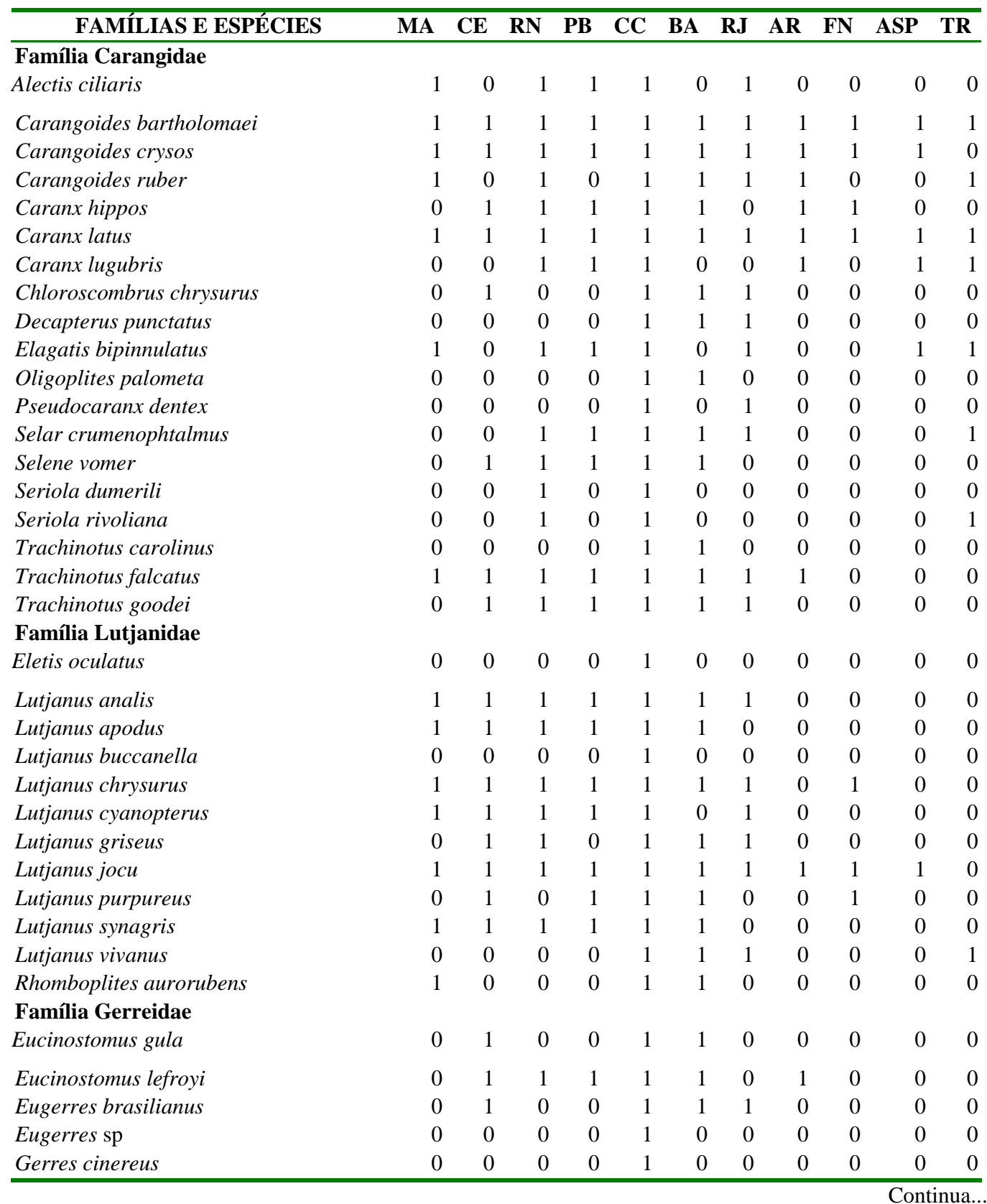


ANEXO 1: REGISTROS BIBLIOGRÁFICOS DA ICTIOFAUNA RECIFAL POR ESTADOS

E ILHAS OCEÂNICAS DO BRASIL. Continuação

\begin{tabular}{|c|c|c|c|c|c|c|c|c|c|c|c|c|}
\hline FAMÍLIAS E ESPÉCIES & MA & $\overline{\mathrm{CE}}$ & $\mathbf{R N}$ & PB & CC & BA & $\mathbf{R J}$ & AR & FN & ASP & TR & $\overline{\mathbf{R}}$ \\
\hline \multicolumn{13}{|l|}{ Família Haemulidae } \\
\hline Anisotremus moricandi & 0 & 1 & 1 & 1 & 1 & 1 & 0 & 0 & 0 & 0 & & 0 \\
\hline Anisotremus surinamensis & 1 & 1 & 1 & 1 & 1 & 1 & 1 & 1 & 1 & 0 & & 1 \\
\hline Anisotremus virginicus & 1 & 1 & 1 & 1 & 1 & 1 & 1 & 1 & 0 & 0 & & 0 \\
\hline Conodon nobilis & 0 & 1 & 0 & 0 & 1 & 1 & 0 & 0 & 0 & 0 & & 0 \\
\hline Haemulon aurolineatum & 1 & 1 & 1 & 1 & 1 & 1 & 0 & 0 & 0 & 0 & & 0 \\
\hline Haemulon parra & 1 & 1 & 1 & 1 & 1 & 0 & 0 & 1 & 0 & 0 & & 0 \\
\hline Haemulon plumieri & 1 & 1 & 1 & 1 & 1 & 1 & 1 & 0 & 0 & 0 & & 0 \\
\hline Haemulon squamipinna & 0 & 1 & 1 & 1 & 1 & 0 & 0 & 0 & 0 & 0 & & 0 \\
\hline Pomadasys corvinaeformis & 0 & 0 & 0 & 0 & 1 & 0 & 0 & 0 & 0 & 0 & & 0 \\
\hline \multicolumn{13}{|l|}{ Família Sparidae } \\
\hline Archosargus rhomboidalis & 0 & 1 & 1 & 1 & 1 & 1 & 1 & 0 & 0 & 0 & & 0 \\
\hline Calamus penna & 0 & 1 & 0 & 1 & 1 & 0 & 1 & 0 & 0 & 0 & & 0 \\
\hline \multicolumn{13}{|l|}{ Família Polynemidae } \\
\hline Polydactylus virginicus & 0 & 0 & 0 & 1 & 1 & 0 & 0 & 0 & 0 & 0 & & 0 \\
\hline \multicolumn{13}{|l|}{ Família Sciaenidae } \\
\hline Bairdiella ronchus & 0 & 0 & 0 & 0 & 1 & 0 & 0 & 0 & 0 & 0 & & 0 \\
\hline Larimus breviceps & 0 & 0 & 0 & 0 & 1 & 1 & 0 & 0 & 0 & 0 & & 0 \\
\hline Equetus lanceolatus & 1 & 1 & 0 & 1 & 1 & 1 & 1 & 0 & 0 & 0 & & 0 \\
\hline Odontoscion dentex & 0 & 0 & 1 & 1 & 1 & 0 & 1 & 0 & 0 & 0 & & 0 \\
\hline Pareques acuminatus & 1 & 1 & 1 & 1 & 1 & 1 & 1 & 0 & 0 & 0 & & 0 \\
\hline \multicolumn{13}{|l|}{ Família Mullidae } \\
\hline Mulloidichthys martinicus & 1 & 0 & 1 & 1 & 1 & 0 & 1 & 1 & 1 & 0 & & 1 \\
\hline Pseudupneus maculatus & 1 & 1 & 1 & 1 & 1 & 1 & 1 & 1 & 1 & 0 & & 1 \\
\hline \multicolumn{13}{|l|}{ Família Pempheridae } \\
\hline Pempheris schomburgki & 1 & 0 & 1 & 1 & 1 & 1 & 1 & 1 & 1 & 0 & & 0 \\
\hline \multicolumn{13}{|l|}{ Família Chaetodontidae } \\
\hline Chaetodon ocellatus & 1 & 1 & 1 & 1 & 1 & 0 & 1 & 1 & 1 & 0 & & 0 \\
\hline Chaetodon striatus & 1 & 1 & 1 & 1 & 1 & 1 & 1 & 0 & 1 & 1 & & 1 \\
\hline \multicolumn{13}{|l|}{ Família Pomacanthidae } \\
\hline Holacanthus ciliaris & 1 & 1 & 1 & 1 & 1 & 1 & 1 & 1 & 1 & 1 & & 0 \\
\hline Holacanthus tricolor & 0 & 1 & 1 & 1 & 1 & 0 & 1 & 0 & 0 & 0 & & 1 \\
\hline Pomacanthus paru & 1 & 1 & 1 & 1 & 1 & 1 & 1 & 1 & 1 & 1 & & 0 \\
\hline \multicolumn{13}{|l|}{ Família Kyphosidae } \\
\hline Kyphosus sectatrix & 1 & 1 & 1 & 1 & 1 & 1 & 0 & 1 & 1 & 1 & & 1 \\
\hline
\end{tabular}


ANEXO 1: REGISTROS BIBLIOGRÁFICOS DA ICTIOFAUNA RECIFAL POR ESTADOS

E ILHAS OCEÂNICAS DO BRASIL. Continuação

\begin{tabular}{|c|c|c|c|c|c|c|c|c|c|c|c|c|}
\hline FAMÍLIAS E ESPÉCIES & MA & $\mathbf{C E}$ & RN & PB & $\overline{\mathrm{CC}}$ & BA & $\mathbf{R J}$ & AR & FN & ASP & TR & \\
\hline \multicolumn{13}{|l|}{ Família Pomacentridae } \\
\hline Abudefduf saxatilis & 1 & 1 & 1 & 1 & 1 & 1 & 1 & 1 & 1 & 1 & & 1 \\
\hline Chromis multilineata & 1 & 1 & 1 & 1 & 1 & 0 & 1 & 1 & 1 & 1 & & 1 \\
\hline Microspathodon chrysurus & 1 & 0 & 1 & 1 & 1 & 1 & 1 & 0 & 0 & 0 & & 1 \\
\hline Stegastes fuscus & 0 & 1 & 1 & 1 & 1 & 1 & 1 & 0 & 0 & 0 & & 0 \\
\hline Stegastes pictus & 1 & 1 & 1 & 1 & 1 & 1 & 1 & 1 & 1 & 0 & & 1 \\
\hline Stegastes variabilis & 0 & 0 & 1 & 1 & 1 & 1 & 1 & 0 & 0 & 0 & & 0 \\
\hline \multicolumn{13}{|l|}{ Família Dactyloscopidae } \\
\hline Dactyloscopus tridigitatus & 0 & 0 & 0 & 1 & 1 & 0 & 0 & 0 & 0 & 0 & 0 & 0 \\
\hline \multicolumn{13}{|l|}{ Família Labridae } \\
\hline Bodianus rufus & 1 & 1 & 1 & 1 & 1 & 1 & 1 & 0 & 1 & 0 & 1 & 1 \\
\hline Clepticus brasiliensis & 1 & 1 & 1 & 1 & 1 & 0 & 1 & 1 & 1 & 1 & & 1 \\
\hline Doratonotus megalepis & 0 & 0 & 1 & 1 & 1 & 1 & 1 & 0 & 0 & 0 & & 0 \\
\hline Halichoeres brasiliensis & 1 & 1 & 1 & 1 & 1 & 1 & 1 & 0 & 0 & 0 & & 1 \\
\hline Halichoeres cyanocephalus & 1 & 1 & 1 & 1 & 1 & 1 & 1 & 1 & 1 & 0 & & 0 \\
\hline Halichoeres maculipinna & 1 & 1 & 1 & 1 & 1 & 1 & 1 & 0 & 0 & 0 & & 1 \\
\hline Halichoeres poeyi & 1 & 1 & 1 & 1 & 1 & 1 & 1 & 0 & 0 & 0 & & 1 \\
\hline Thalassoma noronhanum & 1 & 1 & 1 & 1 & 1 & 1 & 1 & 1 & 1 & 1 & & 1 \\
\hline \multicolumn{13}{|l|}{ Família Scaridae } \\
\hline Sparisoma amplum & 1 & 1 & 1 & 1 & 1 & 0 & 1 & 1 & 0 & 0 & & 1 \\
\hline Sparisoma atomarium & 0 & 0 & 0 & 0 & 1 & 0 & 1 & 0 & 0 & 0 & & 1 \\
\hline Sparisoma axillare & 1 & 1 & 1 & 1 & 1 & 1 & 1 & 1 & 1 & 1 & & 1 \\
\hline Sparisoma frondosum & 1 & 1 & 1 & 1 & 1 & 1 & 1 & 1 & 1 & 1 & & 0 \\
\hline Sparisoma radians & 1 & 0 & 1 & 1 & 1 & 1 & 1 & 0 & 0 & 0 & & 0 \\
\hline Scarus trispinosus & 1 & 0 & 1 & 1 & 1 & 1 & 1 & 0 & 0 & 0 & & 0 \\
\hline Scarus zelindae & 1 & 1 & 1 & 1 & 1 & 1 & 1 & 0 & 0 & 0 & 0 & 0 \\
\hline \multicolumn{13}{|l|}{ Família Trypterigiidae } \\
\hline Enneanectes altivelis & 1 & 0 & 1 & 1 & 1 & 1 & 0 & 1 & 0 & 0 & 1 & 1 \\
\hline \multicolumn{13}{|l|}{ Família Labrisomidae } \\
\hline Labrisomus kalisherae & 0 & 0 & 0 & 1 & 1 & 0 & 1 & 1 & 1 & 0 & 0 & 0 \\
\hline Labrisomus nuchipinnis & 0 & 1 & 1 & 1 & 1 & 0 & 1 & 0 & 0 & 0 & 1 & 1 \\
\hline Malacoctenus delandei & 0 & 0 & 0 & 1 & 1 & 0 & 0 & 0 & 0 & 0 & & 0 \\
\hline Malacoctenus aff triangulatus & 0 & 1 & 0 & 1 & 1 & 1 & 0 & 0 & 0 & 0 & & 0 \\
\hline Paraclinus rubicundus & 0 & 0 & 0 & 1 & 1 & 0 & 0 & 0 & 0 & 0 & & 0 \\
\hline Starksia brasiliensis & 1 & 0 & 1 & 0 & 1 & 0 & 1 & 0 & 0 & 0 & & 0 \\
\hline
\end{tabular}


ANEXO 1: REGISTROS BIBLIOGRÁFICOS DA ICTIOFAUNA RECIFAL POR ESTADOS

E ILHAS OCEÂNICAS DO BRASIL. Continuação

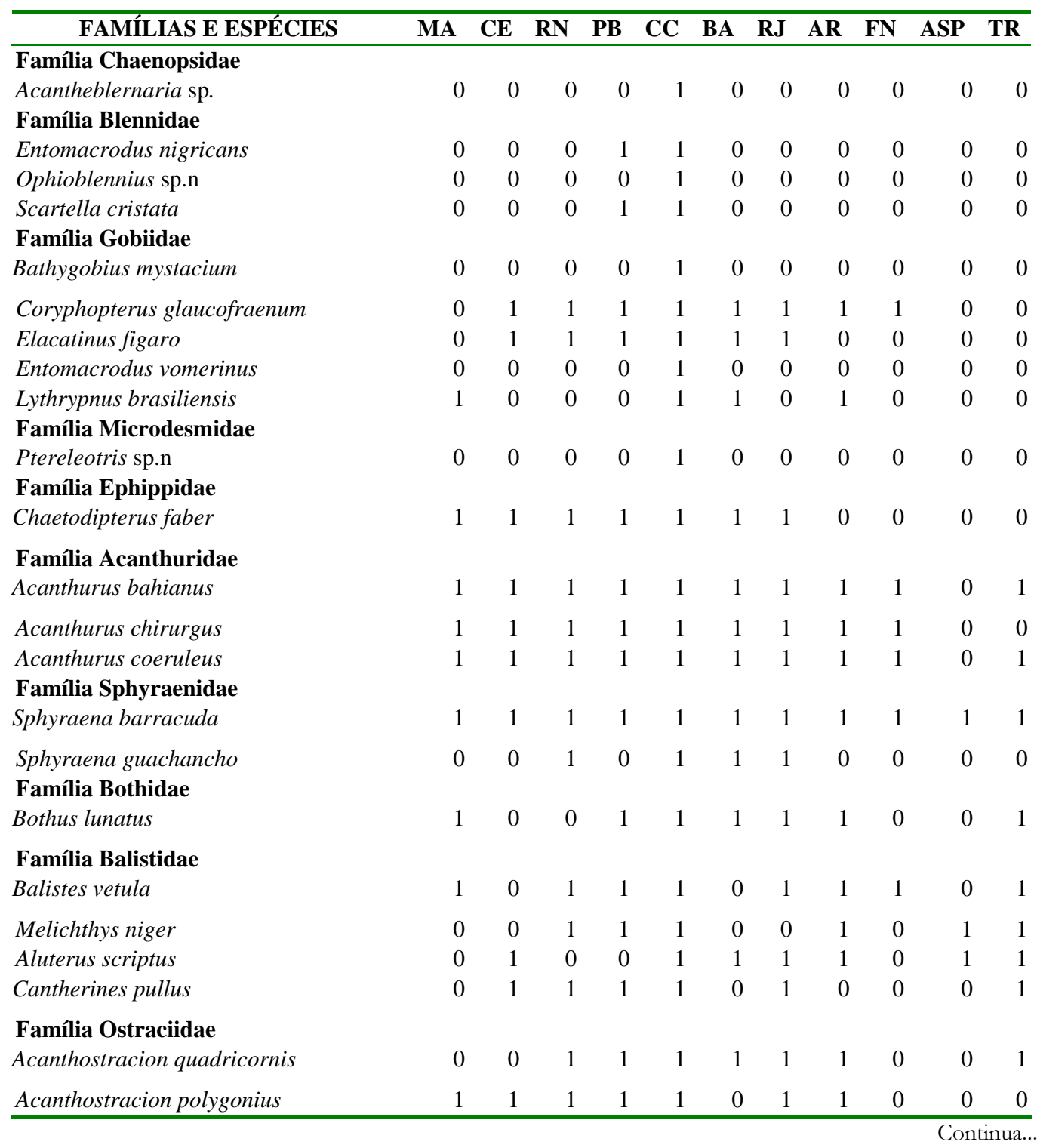


ANEXO 1: REGISTROS BIBLIOGRÁFICOS DA ICTIOFAUNA RECIFAL POR ESTADOS

E ILHAS OCEÂNICAS DO BRASIL. Continuação

\begin{tabular}{|c|c|c|c|c|c|c|c|c|c|c|c|}
\hline FAMÍLIAS E ESPÉCIES & MA & $\overline{C E}$ & $\mathbf{R N}$ & PB & $\mathrm{CC}$ & BA & $\mathbf{R J}$ & AR & FN & ASP & TR \\
\hline \multicolumn{12}{|l|}{ Família Tetraodontidae } \\
\hline Sphoeroides testudineus & 0 & 1 & 1 & 1 & 1 & 1 & 0 & 0 & 0 & 0 & 0 \\
\hline Sphoeroides spengleri & 1 & 0 & 1 & 0 & 1 & 1 & 1 & 0 & 0 & 0 & 0 \\
\hline Canthigasther sp.n & 1 & 1 & 1 & 0 & 1 & 1 & 1 & 1 & 0 & 0 & 0 \\
\hline \multicolumn{12}{|l|}{ Família Diodontidae } \\
\hline Diodon hystrix & 1 & 1 & 1 & 0 & 1 & 0 & 1 & 1 & 1 & 1 & 1 \\
\hline
\end{tabular}

Legenda: Maranhão (MA), Ceará (CE), Rio Grande do Norte (RN), Paraíba (PB), Costa dos Corais (CC), Bahia (BA), Rio de Janeiro (RJ), Atol das Rocas (AR), Fernando de Noronha (FN), Arquipélago São Pedro e São Paulo (ASP) e Trindade (TR). 